\title{
Reliable data transmission: An IoT health-based paradiam
}

\author{
Dalal Abdulmohsin Hammood ${ }^{1}$, Mahmoud Shuker Mahmoud ${ }^{2}$, Ahmed alkhayyat ${ }^{3}$, \\ Hasliza A Rahim ${ }^{4}$, R. Badlishah Ahmad ${ }^{5}$ \\ ${ }^{1}$ Department of Computer Technical Engineering/Middle Technical University (MTU), Iraq \\ ${ }^{2}$ Department of Computer Technical Engineering, Al-Mansour University College, Iraq \\ ${ }^{3}$ Department of Computer Technical Engineering, Islamic University, Iraq \\ ${ }^{4}$ Bioelectromagnetics Research Group (BioEM), Universiti Malaysia Perlis (UniMAP), Malaysia \\ ${ }^{5}$ School of Computer and Communication Engineering, Universiti Malaysia Perlis (UniMAP), Malaysia
}

\begin{abstract}
Article Info
Article history:

Received Feb 9, 2020

Revised Apr 7, 2020

Accepted Apr 21, 2020

Keywords:

CD-TMS

IEEE 802.15.6

IoT

WBAN

ABSTRACT

A WBAN can be spread over a person's body, so; posing, dressing, moving of muscles, degree of heat of bodies as well as weather changes in general affect connections among sensors as well as desired point. Secondly, information collected via every sensor can be connected to a person's course of life; so, it can be considered as significant to guarantee that the collected information ought to be given to desired point competently. In this work, a critical cooperating communicating protocol to WBAN established on information has been suggested, called critical Data Transmission using Transmission Mode Selection (CD-TMS) method established for the architecture of star topology. In the beginning, we performed a studying, also we established a system model can be looked at according to channel los of path, effective transmitting possibility, as well as the out of work time possibility. Thirdly, relaying selecting can be used accompanied by CDTMS, that lets just the most successful relaying take part in cooperating in a spread manner. Simulating as well as numeral outcomes illustrate that CDTMS has the ability to develop the performing of a network under common terms comparing with direct transmission mode (DTM) IEEE 802.15.6 CSMA as well as benchmark.
\end{abstract}

Copyright $\odot 2020$ Institute of Advanced Engineering and Science. All rights reserved.

\section{Corresponding Author:}

Dalal Abdulmohsin Hammood,

Department of Computer Technical Engineering,

Middle Technical University (MTU),

Al Doura 10022, Baghdad, Iraq

Email: dalal.alsaady@eetc.mtu.edu.iq \& dalalmm559@gmail.com

\section{INTRODUCTION}

The IoT has been a developing eco-system which merges durable equipment, gadgets, materialistic things, software, as well as organisms which includes humans and other creatures on a type of networking that makes them able to react, connect, gather as well as reciprocate information. There are various major types of IoT services, from the industrial applications to eHealth care applications. In this research, the smart wearable/implants sensors, machine devices attached on or inside a human body for monitoring in a hospital digital healthcare system are considered, for the purpose of gathering information about the wellness and illness level of a person on heart pulsation, blood pressure, blood sugar level, etc., through sensors on fashion electronics [1-4].

The technologies of WBSN are considered as a technology that is highly imperious and has the ability to be utilized within IoT-based preservation of health pattern [5]. The technology of WBSN may be considered as a set of appliances that are light and of low power, including a transceiving device that is applied for monitoring the important indications. All of the WBSN's sensors for example EEG and ECG are 
able to collect mental indications, besides BT as well as movements. It will then transfer the gathered indications to the coordinator node or $\mathrm{CN}$ on a channel carried by radio or microwave frequencies, or by HBC, that is human body channel to be analyzed and inspected [6]. The patterns of WBSN possess the capability to offer extended health-care of human observation. There will also not be any need to limit their capability or efficacy of moving. Those patterns may be utilized to create a smart and cheap health-care monitoring device which is utilized for diagnostic procedure [7].

Through its widespread development, the IoT will be within the primitive phase and possess a large space to consider various topics such as standards, enumerating resources to the system so that the property of a system is able to lead a growing amount of work, diverseness of a constituent structure, joint definition language, service of specific field detection, as well as integration accompanied by standing systems of Information Technology, amongst others. From past studies, a few discoveries in IoT patterns was established to take care of the wellbeing, for example, safe IoT paradigms on maintenance of health operation on WBSN was presented in [8]. They used reliable crypto-primitives to build dual communicating mechanisms to ensure transmition as well as originating structure authenticating on every sensor of the $\mathrm{CN}$ as well as ridge for network. In [9], the current researches in WBSN area were reviewed accompanied by a certain concentration on the consumption that is not high in power, accurate transmitting, the time interval between the stimulation and response, the transmission speed or the number of bits per second transferred, as well as secureness. In addition to this, the authors considered the needs as well as the WBAN requirements in a conventional e-healthcare frame-work which may discover how such systems are able of obtain efficient communication in the home environment network. A tied mathematical expression for the built-in and builtout channel statistical model that could describe the transmission among transmitter and receiver antennas is presented and studied in [10]. The analysis is conducted according to the 3D digital figure of a human.

In [11], the researches thought of a different design of IoT health maintenance model that is regarded as a safe electronic maintenance of health pattern established on Internet of Things catering to BSNcare. In [12], complete secureness scheme of the dynamic maintenance of health Internet of Things model is suggested. For this study, the researches utilized the impression of smoky tiers in IoT, realizing smooth portability for the smoke which increases the cloud process to the brink of the organization. In [13], the utilization of IoT in the ehealthcare industries are studied and presented, that identifies the digitalization tendency of upcoming study in ehealthcare IoT-based model. In [14], an actual detection tool with solar energy equipped with ground-level capacity transposal representation that enables the utilization of a selfoperating WBSN is explored. Besides that, some mobile app that can be accessed over a network has been designed to show the collected information off a body. In [15], the effect of the energy source allocation as well as small amount of determination for the above the air surrounding, over the maintenance of heath's performing which established on Internet of Things system are being put into account and studied. In the investigation, three particular methods are proposed: determination of power scale, decision of power size and scale, decision of international linking. In [16], a novel Internet of Things aware Smart Hospital System can be presented. The presented system is able to appropriately manage the critical situations. Interoperability stayed a big load for the researchers as well as Internet of Things system improvers. But in [17], a model that can exchange data with unambiguous, shared meaning established on Internet of Things is proposed that is given a semantic interoperability for the heterogeneous IoT device. Doctors can get hold of the sick people of theirs because the gathered data transmitted among one another were explained and communicated in a semantic rationally. In [18], a developed conception is created among cloud computing and IoT: the cloud Internet of Things well-being (CC-IoT) systems. This CC-IoT as well as numerous key integrating issues can be investigated for demonstrating a functional seeing which integrated present mechanism of CC and IoT in apps on maintenance of health. In [19], a confidentiality defender is proposed, that takes care of the data confidentiality that is compiled. The Slepian-Wolf private sharing established on coding may be utilized within Privacy Protector overcoming several kinds of securing mechanisms. In [20], a second kind of fuzzy assisted by ontology recommending regulation to maintenance of health established on Internet of Things is proposed for skillfully observing sick people's bodies. This system greatly improved the predicting accurateness of sick people's situation as well as preciseness rate for medical and nutrition recommendations. In [21], power collecting IoT pattern established on health: to period of out of power possibility reducing throughout the network cooperation of inter-wireless body area has been introduced.

The contributions of this paper are summarized as follows: In the case of the origin to the end route is poorer compared to the basis to relay, collaboration transmittal approach is considered. Most favourable relay is chosen to cooperate together with transmittal collaboration. Collected materials have been considered, whereby analytical data transferred via ccoperative coomunication as well as typical input transferred via DTM.

What remains of the paper can be arranged just like the following: The architecture of WBSN can be depicted with the second section, that includes a couple of small sections in its turn, a WBSN within a network of health established on Internet of Things, as well as novel corporation of inter WBSN besides the 
essential process belongs to suggested protocols. And in the third section, the connection analysis and possibility of out of work period for direct transmission as well as suggested protocol can be looked at. The energy competence for various transmitting scenarios as well as suggested protocols are investigated in Section 4 . The performing as well as outcomes can be presented within the fifth section. In the end, future work and conclusion can be drawn within the sixth section.

\section{WBSN NETWORKS ARCHITECTURE}

WBSN in a Network of well-being established on Internet of Things We can see a novel architecture of a pattern of Internet of things established on health within the Figure 1 that is parted into 4 tiers, where, all tiers belong to the suggested architecture explained in the way mentioned below:

a) Tier of WBSN, tier\#1, within the tier, the sensors could be connected in a direct way to a body of a human or sewed into material (the sensor that can be worn) or instilled in a body of a person. Sensors like those could be EMG, ECG, or EEG etc... The information booked by the sensors can be transferred towards $\mathrm{CN}$ by wireless 802.15.6 standard, then $\mathrm{CN}$ transfers the things that have been transferred via sensors towards the following tier over one of the wires of over the air technology.

b) Smart Interface, tier\#2, within the tier, resourceful tools can be employed, such as cell phones, laptop or tablet. The data are then checked and analyzed, after which the data relocated to tier 3 are chosen over the air communicating technology (In another words BT, cellular Base station or Wi-Fi). The second tier represent a bridge tier that is the link of a WBSN and whereby the physical hardware, transmission media, as well as software applied to link computers and the Internet users, as well as some time placed in WBSN space.

c) The framework of internet tier, tier\#3, the level bridge void among tier\#2 and tier\#4 by departing communication science.

d) Care-Services tier (tier\#4), via this tier, the received data patient server, the Patient server: in this tier, the data are stored, analyzed and sent to the designated service, for example, for urgent matters, health specialist, or family.

There has been more than one sensor spread all around a body in a uniform way for observing the condition of health, and the sensors collected as well as transferred information towards MN or master node $[22,23]$. As a WBAN which can be established on the star topology of the single hop type, all sensors transfer the information of theirs instantly towards the master node. The master node after that transfers the information of theirs towards the monitor node, that can be analyzing the taken information or sending information on the Net towards doctor or clinic [24, 25]. Figure 1 (a) illustrates a sample for a conventional system of a WBAN.

In a WBAN, more than one materialistic environment ought to be taken into account, because the networks can have a configuration in/on a body. As sensors which connected on the different side in bodies that are moveable because of many moves of bodies. So, spaces among sensors and master nodes can be differing. More than one sensor could possess a big space or a connection that lacks of power to master nodes, hence transferring information adequately towards the master node inside a single hop can be hard.

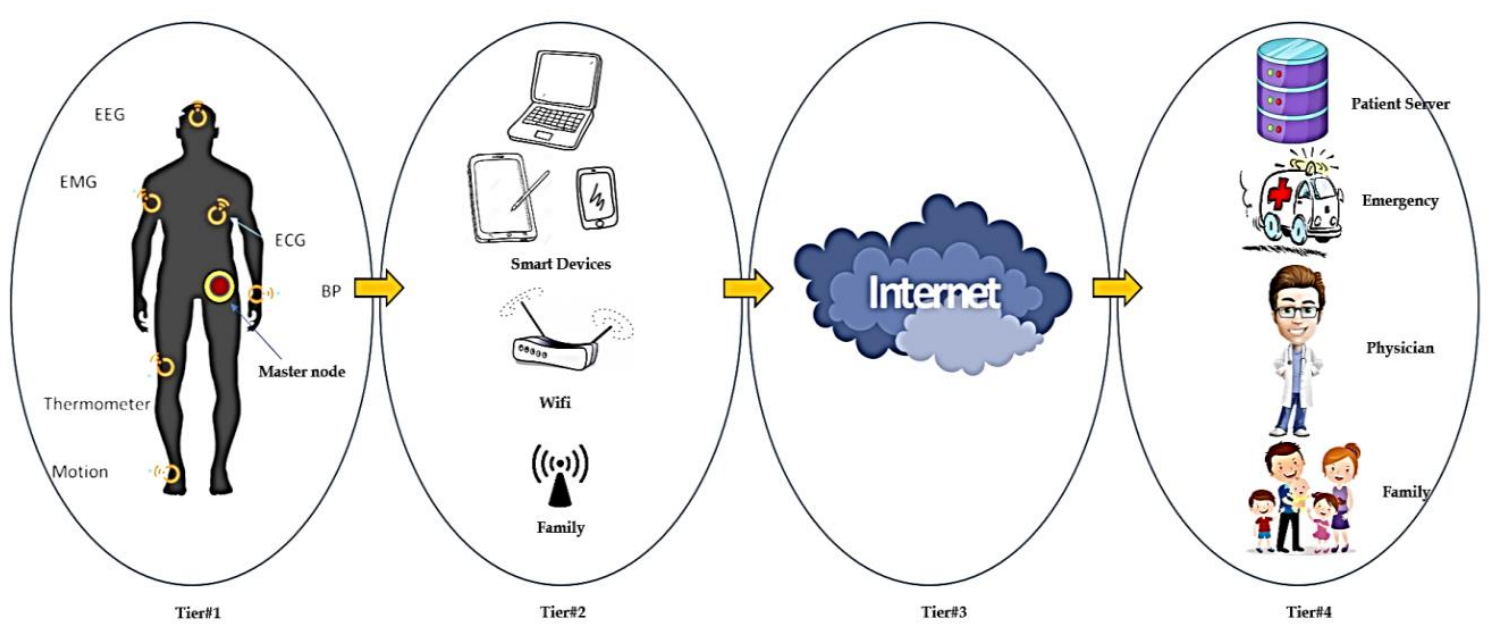

Figure 1. WBSN in IoT-based health networks 
Cooperative communication (CC) can be a great solution for overcoming the issue mentioned above, in another word, single-hop transmittin. Different CC modes can be looked at in the literature in a wide way [26-30]. As ICC can be within the related work as well as it can be briefed as follows: If the master node, or desired point took the packet of information in the right way off source $(S)$, it can transfer acknowledgment, or ACK towards $S$ as well as relay sensor $(R)$ let go the things that are taken off $S$. Or else, it transfers negative acknowledgment, or NACK which permits $R$ transmit again the things that were taken off $S$, but the master nodes let go the things that were taken off $S$ [31, 32]. The cooperating scenario illustrated inside Figure 1 (b). And within the following, the space from $S$ to the master node, $S$ towards $R$ as well as $R$ towards the master node can be indicated as $d_{s d}, d_{s r}$ and $d_{r d}$, in the order given. Besides a connection binds the sensor to the master node as $S-D$. Source as well as relay sensor is bind as $S-R$ and relay sensor is bind to $\mathrm{MN}$ as $R-D$.

\section{LINK AND OUTAGE PROBABILITY ANALYSIS}

The propogation model, the interconnection analysis as well as outage probability of $S-D$ connection is as follows; Where, the signal-to-noise proportion $\left(\gamma_{s d}\right)$ of the $S-D$ link is calculated as:

$$
\gamma_{s d}=\frac{P_{t} \delta_{c}}{P_{N}} K_{s d}
$$

$P_{t}=$ transmission power, $\delta_{c}=$ multiplication of all antennas gained, $P_{N}=$ noise power and $K_{s d}=\mathrm{a}$ complex Gaussian random variable including the unit variance. Therefore, the channel gain $\left|K_{s d}\right|^{2}$ is an exponential dispersed random variable including the mean value, $E\left[\left|K_{s d}\right|^{2}\right]=d_{s d}^{-\alpha}$, where $E$ implies the expectation, $d_{s d}$ signifies the distance of the $S-D$ link, $\alpha$ represents the pathloss signal that interchange from 2 to 6 , that express the inconsistency of the amplitude of a radio indicator on the other end. The transmission degree of $S-D$ link is declared as:

$$
\beta_{s d}=B\left(1+\frac{P_{t} \delta_{c}}{P_{N}} K_{s d}\right)
$$

$B$ represents the transmission route frequency and is stationed to unity. The possibility of the transmission rate is fewer than or equals to the needed transmission rate $\beta_{o}$, is desribed as the outage possibility, where it may be pronounced as [32]:

$$
P_{s d}^{\text {out }}=\left(\beta_{s d} \leq \beta_{o}\right)=1-\exp \exp \left(-\frac{U_{t}}{P_{t} d_{s d}^{-\alpha}}\right)
$$

where, $U_{t}=\frac{P_{N}\left(2^{\beta_{o}}-1\right)}{\delta_{c}}$. Following which, the acceptable transmission probability of the $S-D$ link is demonstrated as

$$
P_{s d}^{s}=1-P_{s d}^{\text {out }}=\exp \exp \left(-\frac{U_{t}}{P_{t} d_{s d}^{-\alpha}}\right)
$$
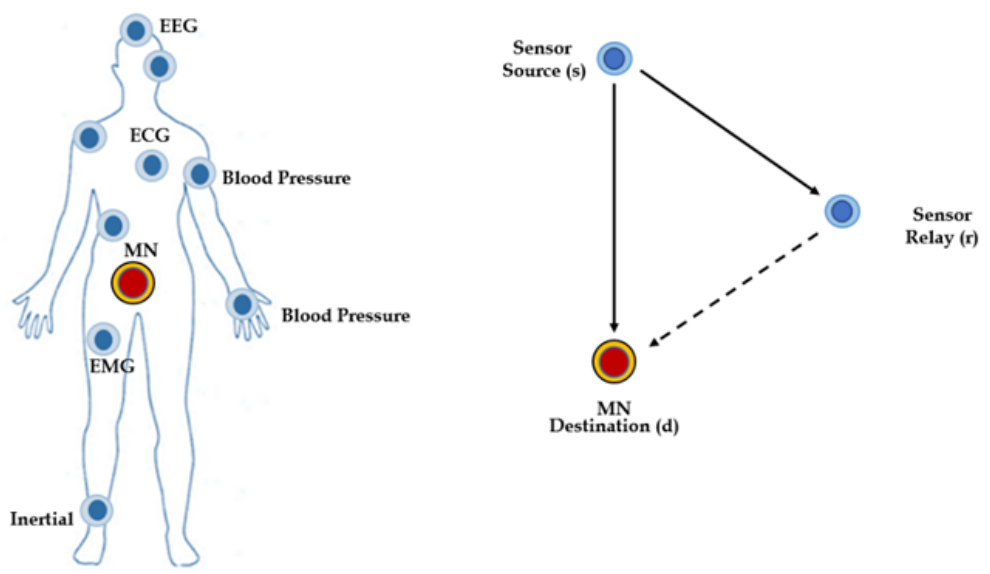

Figure 2. Overall operation of the energy harvesting over inter-WBSN analog network coding (EH-IWANC) 


\section{RELIABLE CRITICAL DATA TRANSMISSION USING TRANSMISSION MODE SELECTION}

\subsection{Proposed method description}

Here, we suggest a competent protocol for transmitting critical information in a trustable way to a WBAN, called Critical Data Transmission utilizing Transmission Mode Selection (CD-TMS). This suggested method functions by taking into account critical information as well as using the concept of mechanical repeat requisation, or ARQ. The CD-TMS possesses two events and can be briefed as below:

The initial event can be expressed as Critical Data Event, indicated to as X, and it takes place as information collected via a sensor can be critical as well as it ought to be transferred to the desired point competently. Critical information transferred throughout an instant path, that can be a resource towards the desired point or throughout the relaying path, that can be a source towards relay, after that relay towards the desired point. Next is Normal Data Event (denoted as Y); happens if the collected data by the sensor is normal or uncritical, thus is transferred straight to the destination by disregarding the relay line.

\subsection{Formulation of the CD-TMS}

Seen previously, the CD-TMS consist of two events where they are declared as

$$
P_{E D-T M S}=P(X)+P(Y)
$$

$P(X)$ represents the probability of the data which is collected by the sensor. The critical data whether transmitted via uninterrupted path or relay path and is calculated as

$$
P(X)=C I . P_{\text {coop }}^{s}
$$

the $C I$ is the critical data index;

$$
C I=1-\left|\frac{\xi_{\min }-\xi_{\max }}{\xi_{\max }}\right|
$$

I. | represents the complete notation, the $\xi_{\max }$ is the most critical data index which is equals to 7 , $\xi_{\min }$ is the least critical data index which differs between 0 to 7 . Thus, $C I$ varies between 0 to 1 , whereby, $\xi_{\min }$ is based on the collected data of the human body, assuming that the data is critical, $\xi_{\min }$ is high, and vice versa. Table 1 displays the probability of the critical data index with another amount of $\xi_{\text {min }}$.

Table 1. The critical data index CI with different values of $\xi_{\text {min }}$

\begin{tabular}{ccc}
\hline$\xi_{\min }$ & $\xi_{\max }$ & $C I$ \\
\hline 0 & 7 & 0 \\
1 & 7 & 0.857 \\
2 & 7 & 0.714 \\
3 & 7 & 0.571 \\
4 & 7 & 0.428 \\
5 & 7 & 0.285 \\
6 & 7 & 0.142 \\
7 & 7 & 1.0 \\
\hline
\end{tabular}

The $P_{\text {coop }}^{s}=\left(1-P_{\text {coop }}^{\text {out }}\right)$ is the successful transmission probability of the coordinated transmission mode with RS and it is calculated as:

$$
\begin{aligned}
P_{c o o p}^{\text {out }}= & P_{s d}^{\text {out }} \cdot P_{s r}^{\text {out }} \cdot P_{o}^{*}+\left(1-P_{s r}^{\text {out }}\right) \\
& P_{s d}^{\text {out }} P_{r d}^{\text {out }}\left(1-P_{o}^{*}\right)
\end{aligned}
$$

where, $P_{s d}^{\text {out }}, P_{s r}^{\text {out }}$ and $P_{r d}^{\text {out }}$ represent $S-D, S-R$ and $R-D$ association is unavailable. Following this, $P_{o}$ represents the probability of the $S-D$ link more than the maximum of the minimum of the $S-R$ and $R-D$ links. It is calculated as:

$$
P_{o}=P\left(K_{s d}>K_{\max }^{s r, r d}\right)=1-P\left(K_{\max }^{s r, r d}>K_{s d}\right)
$$

then,

$$
K_{\max }^{\text {sr, rd }}=\arg \arg \left\{K_{s r_{k}}, K_{r_{k} d}\right\}
$$


where, $K_{\max }^{s r, r d}$ represents the chosen relay index in ordinary relay networks over $k t h$ possible relays, and $K_{s r_{k}}$ as well as $K_{r_{k} d}$ are random variables of $S-R$ and $R-D$ links, respectively. Due to this, the relay is determined according to the channel accomplishments of the relaying links. In consequence, the cumulative distribution function $(\mathrm{CDF})$ of two individual random variables is calculated as:

$$
P_{o}=1-\left(\exp \exp \left(-d_{s r}^{\alpha} K_{s d}\right) \cdot \exp \exp \left(-d_{r d}^{\alpha} K_{s d}\right)\right)
$$

Considering the mean of $P_{o}^{*}$ over $K_{s d}$, thus the mean of the $P_{o}^{*}$ is conveyed

$$
\begin{aligned}
& P_{o}^{*}=1-\int_{0}^{\infty} \exp \exp \left(-K_{s d}\left(\frac{1}{d_{s r}^{-\alpha}}+\frac{1}{d_{r d}^{-\alpha}}\right)\right)\left(\frac{1}{d_{s d}^{-\alpha}}\right) \exp \exp \left(-\frac{1}{d_{s d}^{-\alpha}} K_{s d}\right) d K_{s d}, \\
= & 1-\left(1+\frac{d_{s d}^{-\alpha}}{d_{s r}^{-\alpha}}+\frac{d_{s d}^{-\alpha}}{d_{r d}^{-\alpha}}\right)^{-1}
\end{aligned}
$$

the $1-x \approx \exp (-x)$, then re-interprated (12) as

$$
P_{o}^{*} \approx \exp \exp \left(-\left(1+\frac{d_{s d}^{-\alpha}}{d_{s r}^{-\alpha}}+\frac{d_{s d}^{-\alpha}}{d_{r d}^{-\alpha}}\right)^{-1}\right)
$$

with the assist of (3), replace (13) in (8), $P_{\text {coop }}^{\text {out }}$ is expressed as

$$
\begin{aligned}
& P_{\text {coop }}^{\text {out }}=1-\left(1-\exp \exp \left(-\frac{U_{t}}{P_{t} d_{s d}^{-\alpha}}\right)\right) \boldsymbol{w}_{P_{s d}^{\text {out }}} \cdot\left(1-\exp \exp \left(-\frac{U_{t}}{P_{t} d_{s r}^{-\alpha}}\right)\right)_{\boldsymbol{w}_{P_{s r} \text { out }}} \exp \exp \left(-\left(1+\frac{d_{s d}^{-\alpha}}{d_{s r}^{-\alpha}}+\frac{d_{s d}^{-\alpha}}{d_{r d}^{-\alpha}}\right)^{-1}\right) w_{P_{O}^{*}}+ \\
& \exp \exp \left(-\frac{U_{t}}{P_{t} d_{s r}^{-\alpha}}\right) \omega_{w_{1-P_{s d}^{\text {out }}}}\left(1-\exp \exp \left(-\frac{U_{t}}{P_{t} d_{s d}^{-\alpha}}\right)\right)_{\omega_{P_{s d}^{\text {out }}}}\left(1-\exp \exp \left(-\frac{U_{t}}{P_{t} d_{r d}^{-\alpha}}\right)\right)_{\omega_{P_{r d}^{\text {out }}}}(1- \\
& \left.\exp \exp \left(-\left(1+\frac{d_{s d}^{-\alpha}}{d_{s r}^{-\alpha}}+\frac{d_{s d}^{-\alpha}}{d_{r d}^{-\alpha}}\right)^{-1}\right)\right)_{\omega_{1-P_{o}^{*}}}
\end{aligned}
$$

After that, replace (7) and (14) in (6), critical data event $P(X)$ is expressed as

$$
\begin{aligned}
& P(X)=\left(1-\left|\frac{\xi_{\min }-\xi_{\max }}{\xi_{\max }}\right|\right)_{\omega} C I\left[1-\left(1-\exp \exp \left(-\frac{U_{t}}{P_{t} d_{s d}^{-\alpha}}\right)\right)_{\omega} \omega_{s d}^{\text {out }} .\left(1-\exp \exp \left(-\frac{U_{t}}{P_{t} d_{s r}^{-\alpha}}\right)\right)_{\omega}{ }_{\omega r}^{\text {out }} \exp \exp \left(-\left(1+\frac{d_{s d}^{-\alpha}}{d_{s r}^{-\alpha}}+\right.\right.\right. \\
& \left.\left.\frac{d_{s d}^{-\alpha}}{d_{r d}^{-\alpha}}\right)^{-1}\right) \omega_{P_{0}^{*}}^{*}+\exp \exp \left(-\frac{U_{t}}{P_{t} d_{s r}^{-\alpha}}\right) \omega_{\omega^{1-P_{s d}^{o u t}}}\left(1-\exp \exp \left(-\frac{U_{t}}{P_{t} d_{s d}^{-\alpha}}\right)\right)_{\omega} P_{s d}^{\text {out }}\left(1-\exp \exp \left(-\frac{U_{t}}{P_{t} d_{r d}^{-\alpha}}\right)\right)_{\omega_{r d}^{\text {out }}}(1- \\
& \left.\left.\exp \exp \left(-\left(1+\frac{d_{s d}^{-\alpha}}{d_{s r}^{-\alpha}}+\frac{d_{s d}^{-\alpha}}{d_{r d}^{-\alpha}}\right)^{-1}\right)\right)_{\omega^{1-P_{o}^{*}}}\right]
\end{aligned}
$$

Consequently, noncritical data event is $P(Y)$ and it is interpreted as

$$
P(Y)=(1-C I) \cdot\left(1-P_{s d}^{\text {out }}\right)
$$

Lastly, replace (16) and (15) in (5), the CD-TMS probability is achieved

\section{SIMULATION AND RESULTS DISCUSSION}

Here, the CD-TMS protocol performing which can be submitted in the sections above could be estimated according to effective transmitting possibility, end to end delaying, as well as throughput. Within the simulating here, random topology can be taken into account, as the sensors can be spread in a random way in $3.5 \times 3.5$ square area that is measured in meter, more than one sensor can be fixed inside the area mentioned. The desired point can be placed at $(0,0)$, besides corresponding source sensor placed at $\left(d_{s d}, 0\right)$, furthermore, the relay sensors' number can be differing as well as spread in a random way among desired point and source.

Figure 3 depicts the comparison of the outage probability of DTM, and CD-TMS method for the varying internode distance $S-D$ link. The outage probabilities decline as the radius $S-D$ link also decline. We are able to note the outage probabilities of the CD-TMS which is greater than the outage probability of the DTM. Besides that, the outage probability of the CD-TMS with RS is greater than the outage probability of CD-TMS without RS. The decrease in outage probability of CD-TMS with RS and CD-TMS excluding RS and DTM are 50\% and 99\%, respectively, at $1.8 \mathrm{~m}$. On another note, the outage probability decreament of CD-TMS excluding RS in consideration to DTM appears to be $98 \%$. Figure 4 depicts the association of successful transmission probability of the DTM, as well as CD-TMS protocol for varying internode distance of the $S-D$ link. The successful transmission probabilities at small distance are almost similar. As observed in Figure 4, at big distance, the successful transmission probability of 
the CD-TMS with RS greater than DTM as well as CD-TMS without RS, respectively. The successful transmission probability development of CD-TMS with RS as well as CD-TMS without RS and DTM are 2\% and 18.5\%, respectively, at $2.9 \mathrm{~m}$. In another aspect, successful transmission probability improvement of CD-TMS without RS with respect to DTM was observed as $17 \%$. Figure 5 depicts the comparison of successful transmission probability of the ED-TMS method for varying critical data index values of $\xi_{\text {min }}$. When $\xi_{\text {min }}$ increases, the successful transmission probability elevates as well, and critical data is transferred effectively over the wireless medium. Also observed, is the successful transmission probability of the ED-TMS with RS is greater than ED-TMS by $6 \%$.

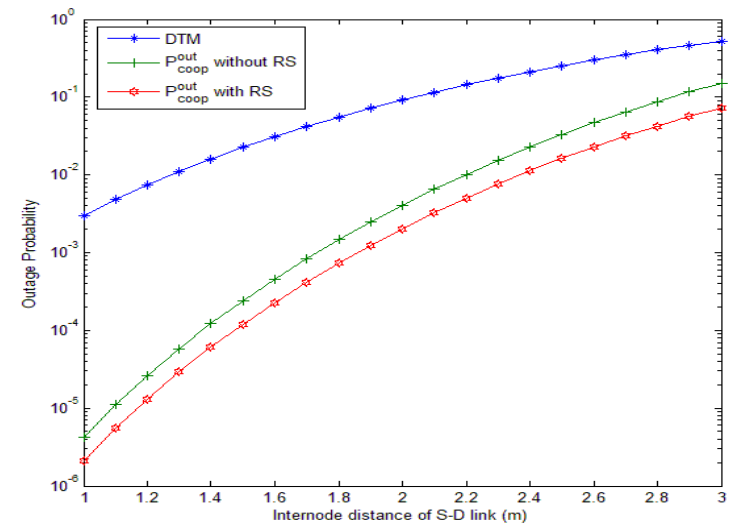

Figure 3. Comparison of outage probability of DTM and CD-TMS with internode distance of $S-D$ link, $\alpha$ is $4, P_{N}=5$, and $\delta_{c}=5$

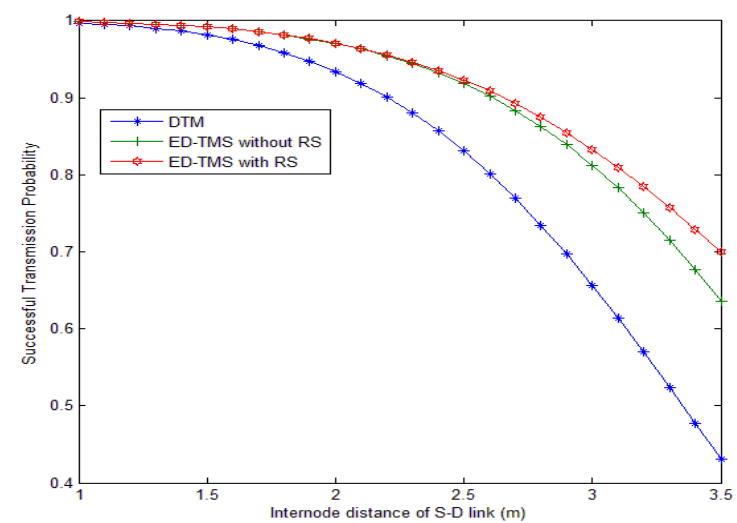

Figure 4. Comparison of successful transmission probability of DTM and CD-TMS with internode distance of $S-D$ link, the $\alpha$ is $4, P_{N}=3, \delta_{c}=5$ and $\xi_{\text {min }}=4$

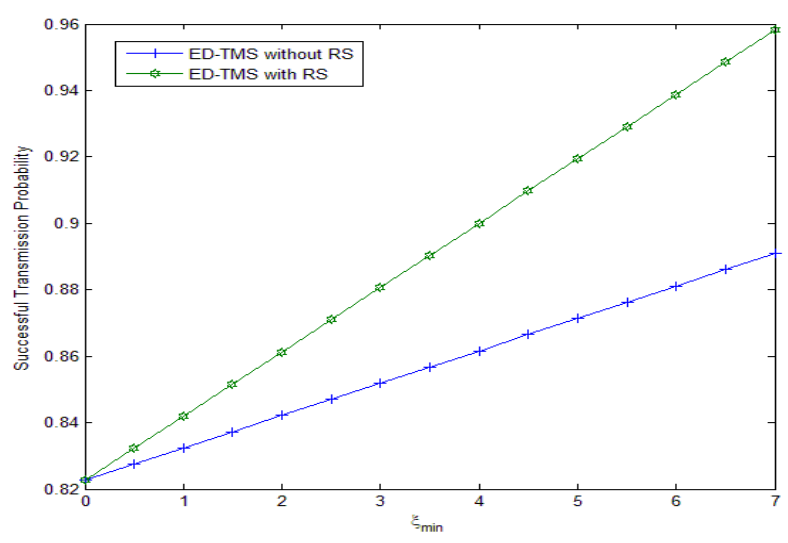

Figure 5. Successful transmission probability of CD-TMS with $\xi_{\min }$. The internode distance $2.5 \mathrm{~m}$ of $S-D$ link, the $\alpha$ is $5, P_{N}=5$, and $\delta_{c}=5$

\section{CONCLUSION}

Communication system shows different kinds of collected data in reference to IEEE 802.15.6 CSMA policy under Rayleigh fading channel, which is CD-TMS, for WBAN. Besides that, a new relay alternative approach along with CD-TMS was discovered. The suggested method elevated the probability of a successful transmission if the collected data were critical, by choosing the direct path or the relay path, however, if the collected data are not critical, the data were transmitted over direct path. As demonstrated above, the CD-TMS may extensively highlight the successful transmission.

\section{REFERENCES}

[1] Yang, G., Xie, L., Mäntysalo, M., Zhou, X., Pang, Z., Da Xu, L., Kao-Walter, S., Chen, Q. and Zheng, L.R., “A health-IoT platform based on the integration of intelligent packaging, unobtrusive bio-sensor, and intelligent medicine box," IEEE transactions on industrial informatics, vol. 10, no. 4, pp. 2180-2191. 
[2] Hassanalieragh, M., et al., "Health monitoring and management using Internet-of-Things (IoT) sensing with cloudbased processing: Opportunities and challenges," In 2015 IEEE International Conference on Services Computing, pp. 285-292, 2015.

[3] A. A. Thabit, et al., "Energy harvesting Internet of Things health-based paradigm: Towards outage probability reduction through inter-wireless body area network cooperation," International Journal of Distributed Sensor Networks, vol. 15, no.10, pp. 1-12, 2019.

[4] D. A. Hammood, et al., "Body-to-Body Cooperation in Internet of Medical Things: Toward Energy Efficiency Improvement," Future internet, vol. 11, no. 11, p. 239, 2019.

[5] Khan RA, Pathan AS., "The state-of-the-art wireless body area sensor networks: A survey," International Journal of Distributed Sensor Networks, vol. 14, no. 4, 2018.

[6] H. Al-Mishmish, et al., "Critical Data-Based Incremental Cooperative Communication for Wireless Body Area Network," Sensors, vol. 18, pp. 3661, 2018.

[7] D. A. Hammood, et al., "Reliable emergency data transmission using transmission mode selection in wireless body area network," Cogent Engineering, vol. 5, no. 1, pp. 198-206, 2018.

[8] Mishra, D., et al., "Smart RF energy harvesting communications: Challenges and opportunities," IEEE Communications Magazine, vol. 53, no. 4, pp. 70-78, 2015.

[9] Varshney L.R., "Transporting information and energy simultaneously," In Information Theory. ISIT 2008. IEEE International Symposium, pp. 1612-1616, 2008.

[10] Wang L., Hu F., Ling Z., Wang B., "Wireless information and power transfer to maximize information throughput in WBAN," IEEE Internet of Things Journal, vol. 4, no. 5, pp. 1663-1670, 2017.

[11] Ghamari, Mohammad, et al., "A survey on wireless body area networks for ehealthcare systems in residential environments," Sensors, vol. 16, no. 6, p. 831, 2016.

[12] Yuehong, Y.I.N., Zeng, Y., Chen, X. and Fan, Y., "The internet of things in healthcare: An overview," Journal of Industrial Information Integration, vol. 1, pp. 3-13, 2016.

[13] Yeh, K. H., "A secure IoT-based healthcare system with body sensor networks," IEEE Access, vol. 4, pp.10288-10299, 2016.

[14] Madhumathi, R.M., Jagadeesan, A. and Kaushik, S., "Healthcare Monitoring System Using Body Sensor Network," In International Conference on Engineering Innovations and Solutions (Iceis-2016), pp. 171-176, 2016.

[15] Moosavi, S.R., Gia, T.N., Nigussie, E., Rahmani, A.M., Virtanen, S., Tenhunen, H. and Isoaho, J., "End-to-end security scheme for mobility enabled healthcare Internet of Things," Future Generation Computer Systems, vol. 64, pp.108-124, 2016.

[16] Luo, E., Bhuiyan, M. Z. A., Wang, G., Rahman, M. A., Wu, J., Atiquzzaman, M., "PrivacyProtector: Privacy-Protected Patient Data Collection in IoT-Based Healthcare Systems," IEEE Communications Magazine, vol. 56, no. 2, 163-168, 2018.

[17] Tao, H., Bhuiyan, M. Z., Abdalla, A. N., Hassan MM, Zain JM, Hayajneh T., "Secured data collection with hardware-based ciphers for iot-based healthcare," IEEE Internet of Things Journal, vol. 6, no. 1, pp. 410-20, 2019.

[18] Hamza R, Yan Z, Muhammad K, Bellavista P, Titouna F., "A privacy-preserving cryptosystem for IoT Ehealthcare," Information Sciences, 2019.

[19] Elhoseny M., Ramírez-González G., Abu-Elnasr O. M., Shawkat S. A., Arunkumar N., Farouk A., "Secure medical data transmission model for IoT-based healthcare systems," IEEE Access, vol. 6, pp. 20596-20608, 2018.

[20] Alkhayyat A., Thabit A. A., Al-Mayali F. A., Abbasi Q. H., "WBSN in IoT Health-Based Application: Toward Delay and Energy Consumption Minimization," Journal of Sensors, 2019.

[21] D. A. Hammood, et al., "An energy-efficient optimization-based scheme for low power devices in wireless body area networks," Journal of Computational and Theoretical Nanoscience, vol. 16, no. 7, pp.2934-2940, 2019.

[22] D. A. Hammood et al., "Enhancement of the Duty Cycle Cooperative Medium Access Control for Wireless Body Area Networks," in IEEE Access, vol. 7, pp. 3348-3359, 2019.

[23] D. A. Hammood, et al., "Reliable emergency data transmission using transmission mode selection in wireless body area network," Cogent Engineering. vol. 5, no. 1, pp. 198-206, 2018.

[24] H. Al-Mishmish, et al., "Critical Data-Based Incremental Cooperative Communication for Wireless Body Area Network," Sensors, vol. 18, pp. 3661, 2018.

[25] A. Alkhayyat, O. Gazi and S. B. Sadkhan, "The role of delay and connectivity in throughput reduction of cooperative decentralized wireless networks," Mathematical Problems in Engineering, vol. 2015, pp. 1-10, 2015.

[26] A. Alkhayyat, "Joint next-hop/relay selection for distributive multihop cooperative networks," Discrete Dynamics in Nature and Society, vol. 2015, pp. 1-10, 2015.

[27] A. Alkhayyat and S. B. Sadkhan, "Bandwidth efficiency analysis of cooperative communication with Reactive Relay Selection," 2018 International Conference on Engineering Technology and their Applications (IICETA), pp. 77-80, 2018.

[28] A. alkhayyat, K. Al Attabi and Q. h. Abbasi, "Single Relay Selection in the Cognitive Cooperative Network: Toward Bandwidth Efficiency Improvement," 2019 4th Scientific International Conference Najaf (SICN), pp. 222-226, 2019.

[29] H. R. Al-Mishmish, et al., "Improvement of Underlay Cooperative Cognitive Networks Bandwidth Efficiency under Interference and Power Constraints," KSII Transactions on Internet and Information Systems (TIIS), vol. 13, no. 11, pp. 5335-5353, 2019.

[30] A. Alkhayyat and N. A. Habeeb, "A Cooperative MAC Aware Network Coding toward Improving Throughput Wireless Body Area Network,” 2019 2nd Scientific Conference of Computer Sciences (SCCS), pp. 182-187, 2019.

[31] A. Alkhayyat and M. S. Mahmoud, "Hybrid Network Coding and Cooperative Communication in WBAN," 2019 2nd International Conference on Engineering Technology and its Applications (IICETA), pp. 79-82, 2019.

[32] A. Alkhayyat and M. S. Mahmoud, "Novel cooperative mac aware network coding under log-normal shadowing channel model in wireless body area network," International Journal on Communications Antenna and Propagation vol. 9, no. 3, pp. 198-206, 2019. 